\title{
Improved Efficiency of Zea mays Agroinoculation with Maize streak virus
}

\author{
D. P. Martin, Ph.D. Student, and E. P. Rybicki, Associate Professor, Microbiology Department, University of Cape \\ Town, Private Bag, Rondebosch, Western Cape, South Africa, 7701
}

\begin{abstract}
Martin, D. P., and Rybicki, E. P. 2000. Improved efficiency of Zea mays agroinoculation with Maize streak virus. Plant Dis. 84:1096-1098.

Agroinoculation is a technique permitting the transmission of geminivirus genomes cloned in Agrobacterium tumefaciens into a wide variety of mono- and dicotyledonous host plants. Most geminiviruses are obligately transmitted by insect vector species under natural conditions; therefore, agroinoculation has greatly simplified the study of this group of viruses. In many cases, agroinoculation has replaced insect transmission, and has been used to compare virulence characteristics among viruses. Here we report on the discovery that, in agroinfectious Maize streak virus constructs, the orientation of cloned viral genomes relative to the Cauliflower mosaic virus 35S (CaMV35S) promoter of the binary cloning vector pBI121 can significantly affect agroinfectivity of the constructs. Rates at which plants became symptomatic were significantly higher when agroinoculating maize seedlings with constructs containing the CaMV35S promoter upstream of the viral replication-associated protein (Rep) gene than when the same viruses were cloned either in the opposite orientation or into a vector without a strong eukaryotic promoter sequence. Plants infected using the construct with Rep cloned downstream of the CaMV35S promoter also displayed more stunting and, in the early stages of the infection, more severe chlorotic streak symptoms.
\end{abstract}

Keywords: agroinfection, mastrevirus, MSV, symptom assessment

Although natural transmission of geminiviruses is exclusively mediated by specific insect vector species, Grimsley et al. (3) devised an alternative means of transmitting geminiviruses into their host plants. This procedure, called agroinfection or agroinoculation, enables a highly effective and controlled infection of host plants with cloned geminivirus genomes and has become extensively used in geminivirus studies.

For the genus Mastrevirus of the family Geminiviridae, it has previously been demonstrated that the efficiency of the agroinoculaton process in Zea mays and other cereal hosts is affected by a number of factors. Thus, while the Agrobacterium sp. and strain used can affect the frequency with which plants become symptomatic (2), the presence in agroinfectious constructs of two long intergenic regions (LIRs) — instead of only one-greatly increases their infectivity (11).

We described a technique whereby transmission of Maize streak virus (MSV) by agroinfection can be used to rapidly and accurately assess MSV resistance of maize genotypes (7). Although the technique could also be applied to the analysis of

Corresponding author: E. P. Rybicki

E-mail: ed@molbiol.uct.ac.za

Accepted for publication 26 June 2000.

Publication no. D-2000-0815-01R

(c) 2000 The American Phytopathological Society
MSV virulence, we observed that agroinfection frequencies and the severity of infection symptoms were influenced by the manner in which agroinfectious constructs had been produced. Agroinfection is potentially a powerful tool for comparing the virulence of cloned viruses; however, it is essential that differences in symptoms can be wholly attributed to differences between the virus genomes being compared.

In this article, we report that efficiencies of agroinfection by MSV clones can be increased by producing constructs with virus genomes cloned into the binary cloning vector, pBI121, such that their replication-associated protein (Rep) genes are downstream of the Cauliflower mosaic virus 35S (CaMV35S) promoter. Although this finding highlights the importance of uniformity in the preparation of agroinfectious constructs to be compared with one another, it also demonstrates a means of enhancing the agroinfectivity of mild virus strains.

\section{MATERIALS AND METHODS}

Virus clones. Complete genomes of four MSV isolates (MSV-A, MSV-B, MSV-C, and MSV-D) have been cloned in this laboratory and, during preliminary studies based on visual assessment of symptoms in sweet corn (cv. Jubilee), have been identified as being very severe (MSV-A, MSVC), moderately severe (MSV-D), and moderate (MSV-B) in their virulence in maize (unpublished data).

Construction of agroinfectious clones and agroinoculation. Entire genomes of the four MSV isolates were all cloned in both orientations into the BamHI site of pUC19 using standard cloning techniques (10). Each of the eight clones was dimerized according to Palmer (8) and cloned into the EcoRI and $\mathrm{XbaI}$ sites of pBI121 (CLONTECH, Palo Alto, CA) to obtain MSV-AI, MSV-AII, MSV-BI, MSV-BII, MSV-CI, MSV-CII, MSV-DI, and MSVDII (Fig. 1). Dimerized MSV-A genomes were additionally cloned in both orientations into the EcoRI and $\mathrm{XbaI}$ sites of pBIN19 (CLONTECH) to obtain MSVAIII and MSV-AIV (Fig. 1). Constructs were transformed into A. tumefaciens C58C1 (pMP90) (4) by the method of An et al. (1). Agroinoculation of 3-day-old seedlings was performed as described previously (7). Each agroinfectious construct was used to infect both sweet corn (highly MSV sensitive; seed obtained from Starke Ayres nursery, Cape Town, South Africa) and PAN6099 (moderately MSV resistant; seed obtained from D. Nowell, PANNAR, Greytown, South Africa). Agroinfection experiments involving each maize genotype-agroinfectious MSV construct combination were repeated at least three times. Agroinfection experiments involving specific constructs were carried out on groups of 14 plants. For each agroinfection experiment, a group of 14 seedlings injected with sterile distilled water served as uninfected controls. Seedlings were grown under near-uniform conditions in a plant growth room maintained at $22^{\circ} \mathrm{C}$ and $80 \%$ relative humidity with $16 \mathrm{~h}$ of light per day.

Analysis of symptoms. Disease severity was measured in terms of (i) percentages of agroinoculated plants that became symptomatic, (ii) percentages of leaf areas covered by chlorotic lesions in symptomatic plants, and (iii) heights of symptomatic plants relative to uninfected control plants (7). The proportion of plants showing symptoms was determined at 3-day intervals between 5 and 14 days post inoculation. For each MSV isolate-plant genotype combination, these measurements were integrated into an infection rate (IR) value calculated as the mean percentage of symptomatic plants observed at the four assessment times. Percentage of leaf area covered by chlorotic lesions in symptomatic plants was estimated for leaves (number 2 through 6) using a microcomputer-based image analysis technique (7). The image analysis protocol required the use of a digital image capture device and analysis software developed in our labora- 
tory (6). The percentage of chlorotic area of leaves 2 and 3 was assessed 15 days after agroinoculation; the percentage of chlorotic area of leaves four, five, and six was assessed 22, 29, and 35 days after agroinoculation, respectively. Heights of symptomatic plants and uninfected control plants were measured as the distance from their coleoptilar nodes to the tip of their fourth leaves, 15 days after agroinoculation. For specific MSV isolate-maize genotype combinations, a value designated $\mathrm{S}$ (representing stunting occurring as a result of infection) was calculated as the mean height of symptomatic plants expressed as a proportion of the mean height of uninfected control plants. The value of 1-S was used as a description of stunting.

\section{RESULTS AND DISCUSSION}

We investigated the effect of MSV cloning orientation in a series of agroinfectious constructs on the symptoms resulting when these constructs were used in agroinfection of highly MSV sensitive (Jubilee) and moderately MSV resistant (PAN6099) maize genotypes. The type I agroinfectious constructs (Fig. 1) containing the four MSV-isolates were, based on IR values, all more infectious than the type II constructs (Fig. 2). Both the degree of stunting 15 days post inoculation and the chlorotic area on leaves 2 and 3 were greater for the type I than for the type II constructs (Figs. 2 and 3). This was probably due to the difference in the frequency with which plants became symptomatic when injected with the two types of construct.

We hypothesized that the different rates at which plants infected with type I and type II constructs became symptomatic was due to either the sense of the singlestranded DNA (ssDNA) transferred during the agroinoculation process or the orientation in the constructs of viral genomes relative to the CaMV35S promoter. Geminiviruses are ssDNA viruses; therefore, it is feasible that the sense of the singlestranded MSV DNA delivered into maize cells by $A$. tumefaciens during agroinfection may influence the infectivity of agroinfectious constructs.

Rep is the only viral gene product required for replication (5) and, at the onset of an infection, it can be assumed that the first viral gene product expressed is Rep (9). There are believed to be two mechanisms by which infectious unit-length genomes are derived from cloned geminivirus DNA following agroinfection. These are known as homologous recombination and replicational release (12) and, as is the case with natural MSV transmission, both require expression of Rep for the initiation of an infection. It is possible that the CaMV35S promoter upstream from the viral Rep gene in type I constructs (Fig. 1) may contribute to expression of Rep. In so doing, the pro- moter may enable earlier or more rapid infection of the maize cells into which $A$. tumefaciens delivers MSV DNA.

To investigate whether it was the position of the CaMV35S promoter relative to the viral Rep gene or the sense of the ssDNA transferred during agroinfection that was responsible for the differences in type I and type II construct infectivity, we cloned MSV-A in both orientations into the
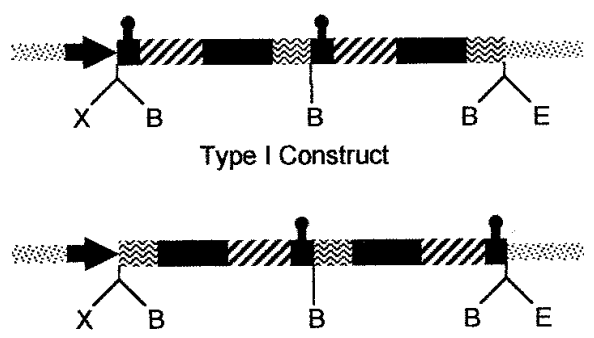

Type II Construct
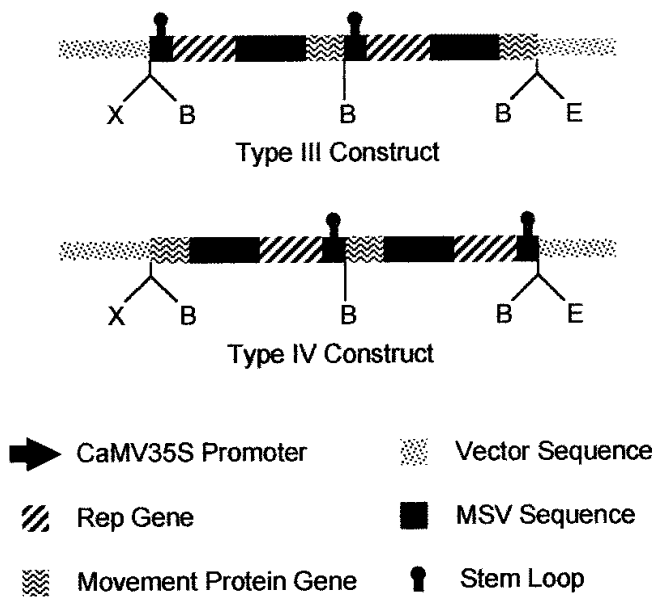

Fig. 1. Types of agroinfectious Maize streak virus (MSV) constructs evaluated in this study. Type I (MSV-AI, MSV-BI, MSV-CI, and MSV-DI) and type II (MSV-AII, MSV-BII, MSV-CII, and MSVDII) constructs are tandemly dimerized full-length MSV genomes cloned in opposite orientations into the XbaI and EcoRI sites of pBI121. Type III (MSV-AIII) and type IV (MSV-AIV) constructs are tandemly dimerized full-length MSV genomes cloned in opposite orientations into the $X b a \mathrm{I}$ and $E c o$ RI sites of pBIN19. Letters B, E, and X represent BamHI, EcoRI, and XbaI restriction sites, respectively.

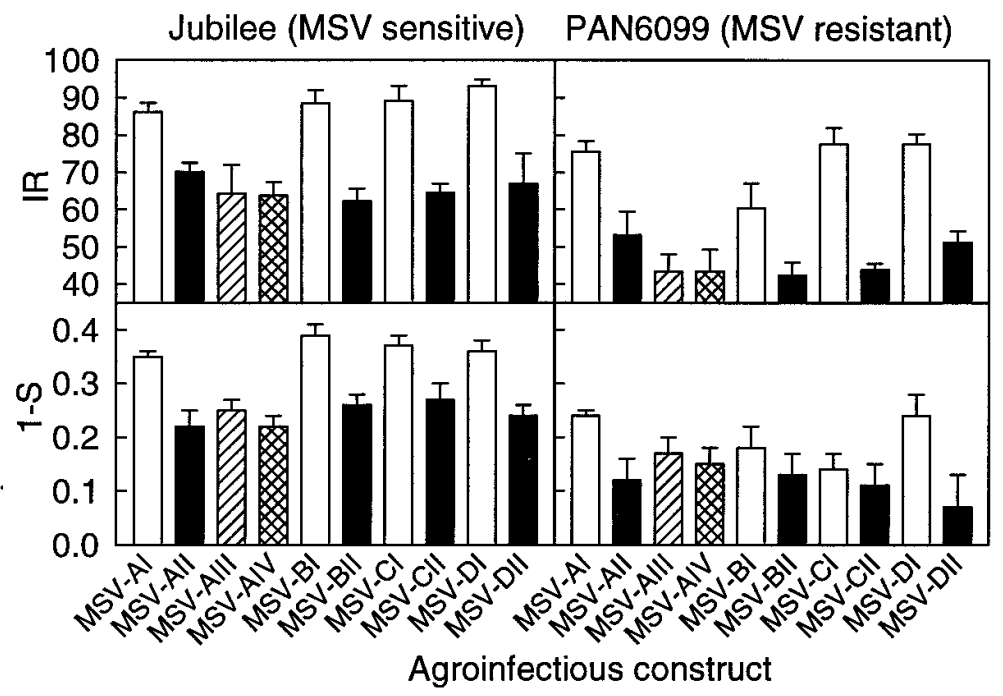

Fig. 2. Relative rates at which plants became symptomatic (IR) and the amount of stunting symptomatic plants exhibited (1-S) when infected with different agroinfectious constructs. Maize streak virus (MSV) type I constructs (MSV-AI, MSV-BI, MSV-CI, and MSV-DI) are represented by white bars, type II constructs (MSV-AII, MSV-BII, MSV-CII and MSV-DII) by black bars, the type III construct (MSV-AIII) by hatched bars, and the type IV construct (MSV-AIV) by crosshatched bars. Error bars represent $95 \%$ confidence intervals of the mean. 


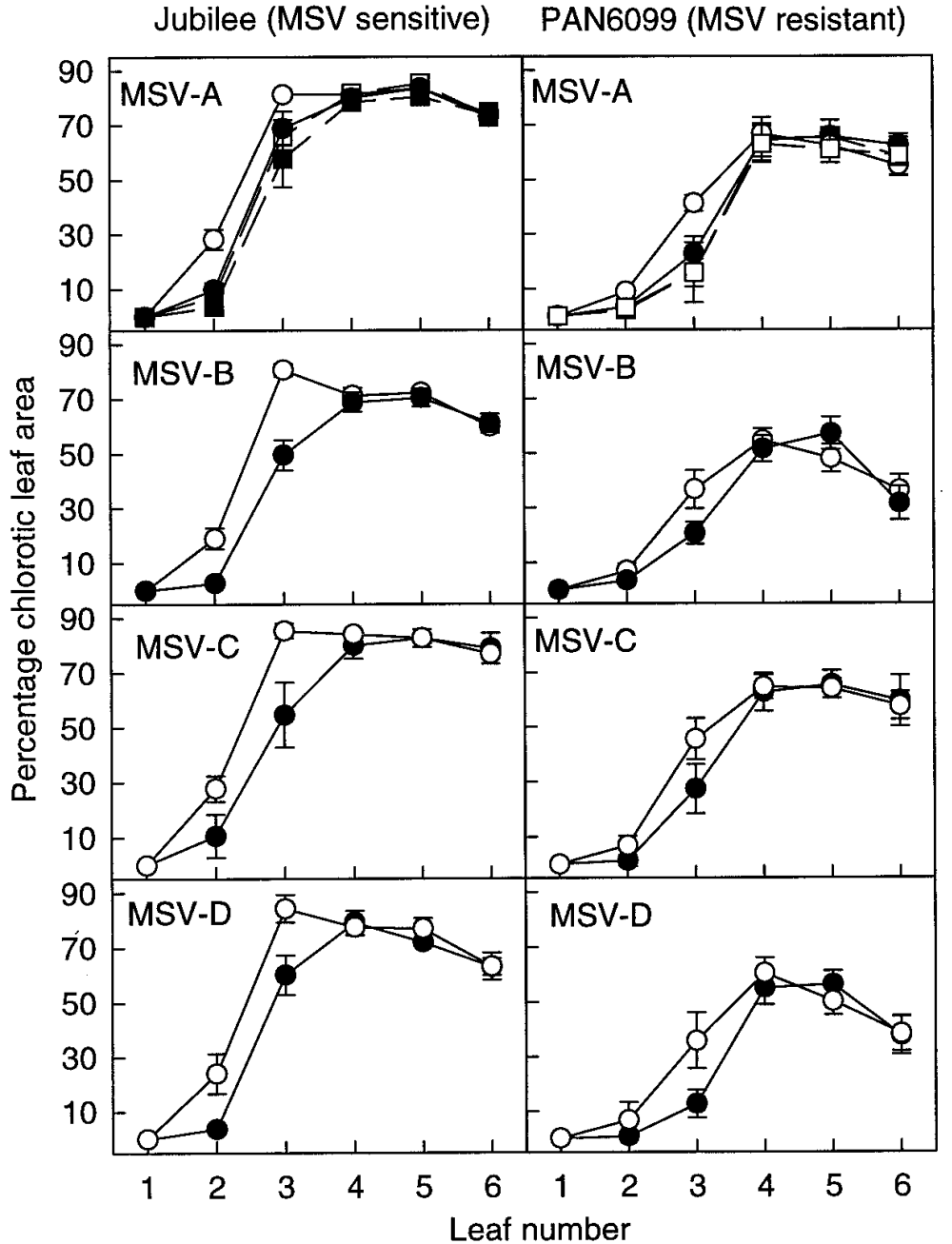

Fig. 3. Percentage of chlorotic area recorded on leaves 2 through 6 of two maize genotypes (Jubilee and PAN6099) agroinoculated with the type I $(\bigcirc)$ and type II $(\bullet)$ agroinfectious constructs. Maize streak virus (MSV) type III and type IV constructs, MSV-AIII ( $\square$ ) and MSVA-IV (ロ), respectively, are represented in the MSV-A panel using broken lines. Error bars on either side of symbols represent $95 \%$ confidence intervals of the mean.

second binary cloning vector pBIN19. Except for the absence of the CaMV35S promoter sequence, the resulting type III (MSV-AIII) and type IV (MSV-AIV) constructs resembled MSV-AI and MSV-AII, respectively (Fig. 1). In both maize genotypes, MSV-AIII and MSV-AIV were not significantly different from one another in infectivity, the degree of stunting they induced (Fig. 2), or the chlorotic areas they produced on leaves 2 through 6 (Fig 3). There were also no significant differences in infectivity or virulence between these constructs and the MSV-AII construct. It is, therefore, unlikely that the sense of the transferred virus ssDNA is responsible for the different type I and type II construct infectivities. Rather, it appears to be the presence of the CaMV35S promoter upstream from the Rep gene in the type I constructs that is responsible for their greater infectivity.
These results indicate that the infectivity, and therefore the apparent virulence, of different geminiviruses introduced into plants by agroinfection can be influenced by the manner in which agroinfectious constructs are produced. This further suggests that, in studies employing agroinfection to compare the virulence of geminivirus isolates, it may be important that all constructs be produced in a uniform manner. If rates at which plants become symptomatic, stunting, and percentage of chlorotic area on the first leaves showing symptoms are to be used as measures of infectivity or virulence, considering potential vector effects is essential. Of all the measures of virulence we examined, the only ones we found not to be influenced by orientation in pBI121 were chlorotic areas that occurred on leaves 4,5 , and 6 . We recommend that, for comparative virulence studies employing agroinfection, whenever different cloning orientations or cloning vectors have been used and there is uncertainty regarding the effects of these differences, rates at which plants become symptomatic, stunting, and chlorotic area on the first few leaves not be used as reliable measures of virulence.

Ways of manipulating agroinfectious clones to enhance their infectivity could greatly aid the study of mild geminivirus isolates. We are currently making use of type I constructs to achieve enhanced infection frequencies when studying severely defective MSV recombinants and mutants in maize.

\section{ACKNOWLEDGMENTS}

We thank J. S. Passmore for proofreading this manuscript, the Foundation for Research Development for providing funding for this research, and D. Nowell for generously providing seed.

\section{LITERATURE CITED}

1. An, G., Ebert, P. R., Mitra, A., and Ha, S. B. 1988. Binary vectors. Pages $87-88$ in: Plant Molecular Biology Manual. S. B. Gelvin and R. A. Schilperoort, eds. Kluwer Dordrecht, The Netherlands.

2. Boulton, M. I., Buchholz, W. G., Marks, M. S., Markham, P. G., and Davies, J. W. 1989. Specificity of Agrobacterium-mediated delivery of maize streak virus DNA to members of the Graminae. Plant Mol. Biol. 12:31-40.

3. Grimsley, N., Hohn, T., Davies, J. W., and Hohn, B. 1987. Agrobacterium-mediated delivery of infectious maize streak virus in maize plants. Nature 325:177-179.

4. Koncz, C., and Schell, J. (1986) The promoter of $T_{L}$-DNA gene 5 controls the tissue-specific expression of chimaeric genes carried by a novel type of Agrobacterium binary vector. Mol. Gen. Genet. 204:383-396

5. Lazarowitz, S. G., Pinder, A. J., Damstgeet, V. D., and Rogers, S. G. 1989. Maize streak virus genes essential for systemic spread and symptom development. EMBO J. 8:10231032.

6. Martin, D. P., and Rybicki, E. P. 1998. Microcomputer based quantification of maize streak virus symptoms in Zea mays. Phytopathology 88:422-427.

7. Martin, D. P., Willment, J. A., and Rybicki, E. P. 1999. Evaluation of maize streak virus pathogenicity in differentially resistant Zea mays genotypes. Phytopathology 89:695-700.

8. Palmer, K. E. 1997. Investigations into the use of Maize streak virus as a gene vector. Ph.D. thesis, University of Cape Town, South Africa.

9. Palmer, K. E., and Rybicki, E. P. 1998. The molecular biology of Mastreviruses. Adv. Virus Res. 50:183-234.

10. Sambrook, J., Fritsch, E. F., and Maniatis, T. 1989. Molecular Cloning: A Laboratory Manual. 2nd ed. Cold Spring Harbor Laboratory Press, Cold Spring Harbor, New York.

11. Schnippenkoetter, W. H. 1998. The use of agroinfectious clones to investigate recombination between distinct maize streak virus strains. Ph.D. thesis, University of Cape Town, South Africa.

12. Stenger, D. C., Revington, G. N., Stevenson, M. C., and Bisaro, D. 1991. Replicational release of geminivirus genomes from randomly repeated copies: evidence for rolling circle replication of a plant viral DNA. Proc. Natl. Acad. Sci. USA 88:8029-8033. 